\title{
Clinorotation Affects Induction of the Heat Shock Response in Arabidopsis thaliana Seedlings
}

\author{
Liudmyla Y. Kozeko ${ }^{1}$, Denis D. Buy ${ }^{2}$, Yaroslav V. Pirko², Yaroslav B. Blume ${ }^{2}$, and Elizaveta L.
} Kordyum $^{1}$

\begin{abstract}
${ }^{1}$ M.G. Kholodny Institute of Botany of the National Academy of Sciences of Ukraine, Ukraine, 01601 Kyiv, Tereschenkivska st. 2; ${ }^{2}$ Institute of Food Biotechnology and Genomics of the National Academy of Sciences of Ukraine, Ukraine, 04123 Kyiv, Osipovskogo st. $2 a$
\end{abstract}

\begin{abstract}
Clinorotation used to simulate microgravity effects in ground-based experiments is considered as a mild stress factor for plants. We have assumed that it might influence the plant tolerance to other stressful factors. To test this, Arabidopsis thaliana seedlings were grown on a horizontal clinostat (2 rpm) or under stationary conditions (control), and then were subjected to heat treatment. The kinetics of gene expression of cytosolic HSP70s and HSP90s during exposure to $37^{\circ} \mathrm{C}$ for $0.5-2 \mathrm{~h}$ was examined by RT-qPCR to estimate level of the heat shock reaction. It was shown that clinorotation caused the minor increase in transcript abundance of five AtHSP70s and AtHSP90-1 under normal temperature, as well as a faster onset and enhancement of their induction during heat shock. The heat tolerance was evaluated as a function of seedling survival after exposure to $45^{\circ} \mathrm{C}$ for $45 \mathrm{~min}$. Seedlings grown under clinorotation were determined to withstand heat treatment better than seedlings grown under stationary conditions. The obtained data support the assumption that clinorotation may provide
\end{abstract}

Key words: Clinorotation; Stress; Heat Shock Proteins; Thermotolerance; Arabidopsis thaliana

Correspondence to: Liudmyla Y. Kozeko

M.G. Kholodny Institute of Botany

NAS of Ukraine

Tereschenkivska st. 2, Kyiv, 01601, Ukraine

Telephone: +380442723236

E-mail: liudmyla.kozeko@gmail.com cross-protection of plants against fluctuations in environmental conditions.

\section{INTRODUCTION}

Steady growing of plants as an autotrophic link of Life Support Systems is one of the key issues for long-term manned space missions (Ferl et al., 2002; Wheeler, 2010). Their ability to cope with spaceflight environments is thus of critical importance. It has been shown that microgravity, an unusual factor for plants, does not prevent their growth and development but causes changes in structural and functional organization of cells, and is considered as a mild stressor (Medina et al., 2011; Kordyum, 2014). However, its influence on the development of adaptive reaction to other unfavorable factors is not well understood. To study effects of reduced gravity in plants in ground-based experiments, we used clinorotation. This study is focused on how clinorotation might influence a conserved reaction of cells to elevated temperatures, known as the heat shock response. A key component of this response is upregulation of heat shock proteins (HSP) (Vierling, 1991; Sørensen et al., 2003; Wang et al., 2004). Functioning as molecular chaperones, they protect and reactivate protein structures affected by stress events.

It has been found that spaceflight factors, including microgravity, activate HSP expression in plant cells (Paul et al., 2005, 2012; Zupanska et al., 2013; Hausmann et al., 2014). In our previous work, some temporary increase in the 
HSP70 and HSP90 levels in pea seedlings for the first hours of clinorotation was determined (Kozeko and Kordyum, 2007). In addition, seedlings grown under permanent rotation showed a perceptible increase in HSP induction in response to high temperatures, when compared to seedlings grown motionlessly (Kozeko, 2008).

Numerous investigations have confirmed the importance of HSPs in plant tolerance to heat, as well as to a range of other stressful factors (Vierling, 1991; Sørensen et al., 2003; Wang et al., 2004). Moreover, their involvement in cross-protection between different types of stress has been examined (Banti et al., 2008; Montero-Barrientos et al., 2010).
In the present work, we have studied the influence of clinorotation on gene expression of HSP70 and HSP90 during the following heat shock treatment, as well as thermotolerance in Arabidopsis thaliana seedlings. HSP70s are considered as the principal stress-induced HSP family in many species (Sørensen et al., 2003). HSP90s also respond to damaging factors (Milioni andHatzopoulos, 1997; Wang et al., 2004). In A. thaliana, there are five homologues of HSP70s (DnaK subfamily) and four homologues of HSP90s that are localized to the cytosol. Among these AtHSP70-4, AtHSP70-5, and AtHSP90-1 are highly induced by heat (Milioni and Hatzopoulos, 1997; Lin et al., 2001; Sung et al., 2001). Refer to Table 1 for gene names and AGI codes.

Table 1. Primers of target genes used for RT-qPCR.

\begin{tabular}{lll}
\hline Gene name & AGI code & Primer sequences (5’ to 3’) \\
\hline AtHSP70-1 & AT5G02500 & $\begin{array}{l}\text { F: AAACCCTAGCCGCCTTATTC } \\
\text { R: GATAGCTGGTCCTTCTCTTTAC }\end{array}$ \\
AtHSP70-2 & AT5G02490 & $\begin{array}{l}\text { F: AGCTTGTGAGAGAGCAAAGAG } \\
\text { R: ACGGGTGATTGGAGAATAGA }\end{array}$ \\
AtHSP70-3 & AT3G09440 & $\begin{array}{l}\text { F: GACATTAGTGGAAACCCGAGAG } \\
\text { R: GTCTGAGCCGTAGATGACAAAG }\end{array}$ \\
AtHSP70-4 & AT3G12580 & $\begin{array}{l}\text { F: AGGGCACGAACAAAGGACAACAAC } \\
\text { R: TCAGCCGCACATTCAGGATACCA }\end{array}$ \\
AtHSP70-5 & AT1G16030 & $\begin{array}{l}\text { F: GGAGCTATCTCTGGGCTTAATG } \\
\text { R: GGCCTTCGTACCCTTCTATC }\end{array}$ \\
AtHSP90-1 & AT5G52640 & $\begin{array}{l}\text { F: GTTACCCTATCTACCTTTGGACCG } \\
\text { R: CTGCTTGTTGATGAGTCCCAC }\end{array}$ \\
AtHSP90-2, & AT5G56030 & To detect mRNA of three genes in total: \\
AtHSP90-3, & AT5G56010 & F: GCTACCCAATCTCTCTCTGGATT \\
AtHSP90-4 & AT5G56000 & R: GTACTCCTCCTTGTTGATCTCCTC \\
AtUBQ5 & AT3G62250 & F: AACCCTTGAGGTTGAATCATCC \\
& R: GTCCTTCTTCTGGTAAACGT \\
\hline
\end{tabular}

\section{MATERIALS AND METHODS}

\section{Plant Material, Growth Conditions, and Heat Treatments}

Arabidopsis thaliana (L.) Heynh. ecotype Columbia (Col-0) seeds were sterilized with $70 \%$ ethanol and hypochlorite solution $(3 \% \mathrm{Cl})$, washed five times with sterile water, incubated in the dark at $4^{\circ} \mathrm{C}$ for $2 \mathrm{~d}$, and plated with equal spacing on germination medium (0.5 Murashige and Skoog mineral salt complex, $1 \%$ sucrose, $0.8 \%$ agar) in Petri plates. Seeds were allowed to germinate and grow on a horizontal clinostat (2 rpm) or under stationary conditions (control) at $22 \pm 1^{\circ} \mathrm{C}$, photoperiod of $16 \mathrm{~h} \mathrm{light/} / 8 \mathrm{~h}$ dark, and light intensity $\sim 110 \mu \mathrm{mol} \mathrm{m} \mathrm{m}^{-2} \mathrm{~s}^{-1}$. After $12 \mathrm{~d}$ of growth, rotated and control seedlings were subjected to heat treatments.

For seedling survival assay, plates with 12d-old seedlings, grown as described above, were 
exposed to $45^{\circ} \mathrm{C}$ for $45 \mathrm{~min}$. Following the heat treatment, seedlings were returned to the stationary conditions and their survival was recorded for the next $6 \mathrm{~d}$ of incubation. The number of viable seedlings that were still green and generated new leaves was quantified to determine the survival rate. This assay was also performed for 5-d-old seedlings.

For analysis of gene expression, 12-d-old seedlings grown, as described above, were exposed at $37^{\circ} \mathrm{C}$ for $0.5,1$, and $2 \mathrm{~h}$ and then frozen at $-70^{\circ} \mathrm{C}$. Three independent experiments were performed for each experimental condition.

\section{RT-qPCR}

Total RNA was extracted from seedlings using innuPREP Plant RNA Kit (Analytik Jena). $1 \mu \mathrm{g}$ of each sample was reverse transcribed into cDNA using RevertAid First Strand cDNA synthesis Kit (ThermoSci) with oligo(dT)18 primer. Real-time amplification was performed using the Maxima SYBR Green/Fluorescein qPCR Master Mix (Thermo Scientific) and the iQ5 amplifier (Bio-Rad). The following PCR program was employed: $95^{\circ} \mathrm{C}$ for $2 \mathrm{~min}$, then 35 cycles of $95^{\circ} \mathrm{C}$ for $20 \mathrm{~s}, 60^{\circ} \mathrm{C}$ for $30 \mathrm{~s}$, and $72^{\circ} \mathrm{C}$ for $40 \mathrm{~s}$. UBQ5 was used as the reference gene for all assays. Primer pairs, as outlined in Table 1 , were designed using IDT PrimerQuest Tool (https://eu.idtdna.com/Primerquest/Home/Index) The conserved sequences of highly homologous AtHSP90-2, AtHSP90-3, and AtHSP90-4 were used to design the common primers for them. Specificity of each primer pair and quality of RT-qPCR products was checked by melting curve analysis and agarose gel electrophoresis. Quantity of each transcript was measured in three independent biological samples with three analytical replicates. UBQ5 expression was used to normalize the transcript levels in each sample. Relative expression of each gene was calculated by the comparative $\Delta \Delta \mathrm{Ct}$ method relatively to the transcript level of the control at $22^{\circ} \mathrm{C}$.

\section{Data Analysis and Statistics}

For data analysis, the mean and standard deviation were calculated for each group. A two-way ANOVA was used to determine significant differences for the effects of clinostat rotation and high temperature on transcript levels $(\mathrm{p}<0.05)$. For the seedling survival and effect of clinostat rotation on HSP expression at normal temperature, the means for the control and experimental groups were compared using Student's t-test $(\mathrm{p}<0.05)$.

\section{RESULTS}

In order to test whether clinorotation influences the heat shock response, seedlings grown under stationary conditions (control) or clinostat rotation for $12 \mathrm{~d}$ were exposed to $37^{\circ} \mathrm{C}$ for 0.5-2 h. Levels of mRNAs of cytosolic HSP70s and HSP90s in seedlings were quantified using RT-qPCR. We used one set of primers for AtHSP90-2, AtHSP90-3, and AtHSP90-4, since their sequences are highly homologous and we could not synthesize specific primers for each gene. In addition, these HSP90s are known to show similar pattern of gene expression at both normal and high temperatures (Milioni and Hatzopoulos, 1997). Therefore, we measured the total mRNA level of these three genes to characterize gene expression of constitutive cytosolic HSP90s.

Figure 1 shows that exposure to $37^{\circ} \mathrm{C}$ resulted in rapid induction of HSP genes in control and rotated seedlings, but with differences in magnitudes of their expression. The mRNA level of constitutive AtHSP70-1 steadily increased at a low rate over the duration of heat exposure, and only a tendency to its enhancement after clinorotation was observed. Induction of constitutive AtHSP70-2, AtHSP703 , as well as AtHSP90-2, AtHSP90-3, and AtHSP90-4 (in total) reached the maximum levels at $1 \mathrm{~h}$ of heat treatment, while thereafter the mRNA amounts declined. At the same time, a higher expression rate for AtHSP70-2 and AtHSP70-3 and, conversely, a tendency to decline for expression of HSP90s were determined in rotated seedlings, when compared to control ones. Inducible AtHSP70-4, AtHSP70-5, and AtHSP90-1 showed strong induction by $30 \mathrm{~min}$ of heat treatment, its significant increase within the next $30 \mathrm{~min}$ and a steady high level for the second hour. After clinorotation, the induction of these genes occurred faster and reached a higher level, especially for AtHSP70-4 and AtHSP70-5.

As illustrated in Figure 2, comparison of the transcript abundance in rotated and control seedlings at $22 \pm 1^{\circ} \mathrm{C}(0 \mathrm{~h}$ of heat exposure) showed an increase of 2- to 7-fold under clinorotation, with the exception of constitutive HSP90s.

The heat tolerance of seedlings was evaluated as a function of their survival after an 

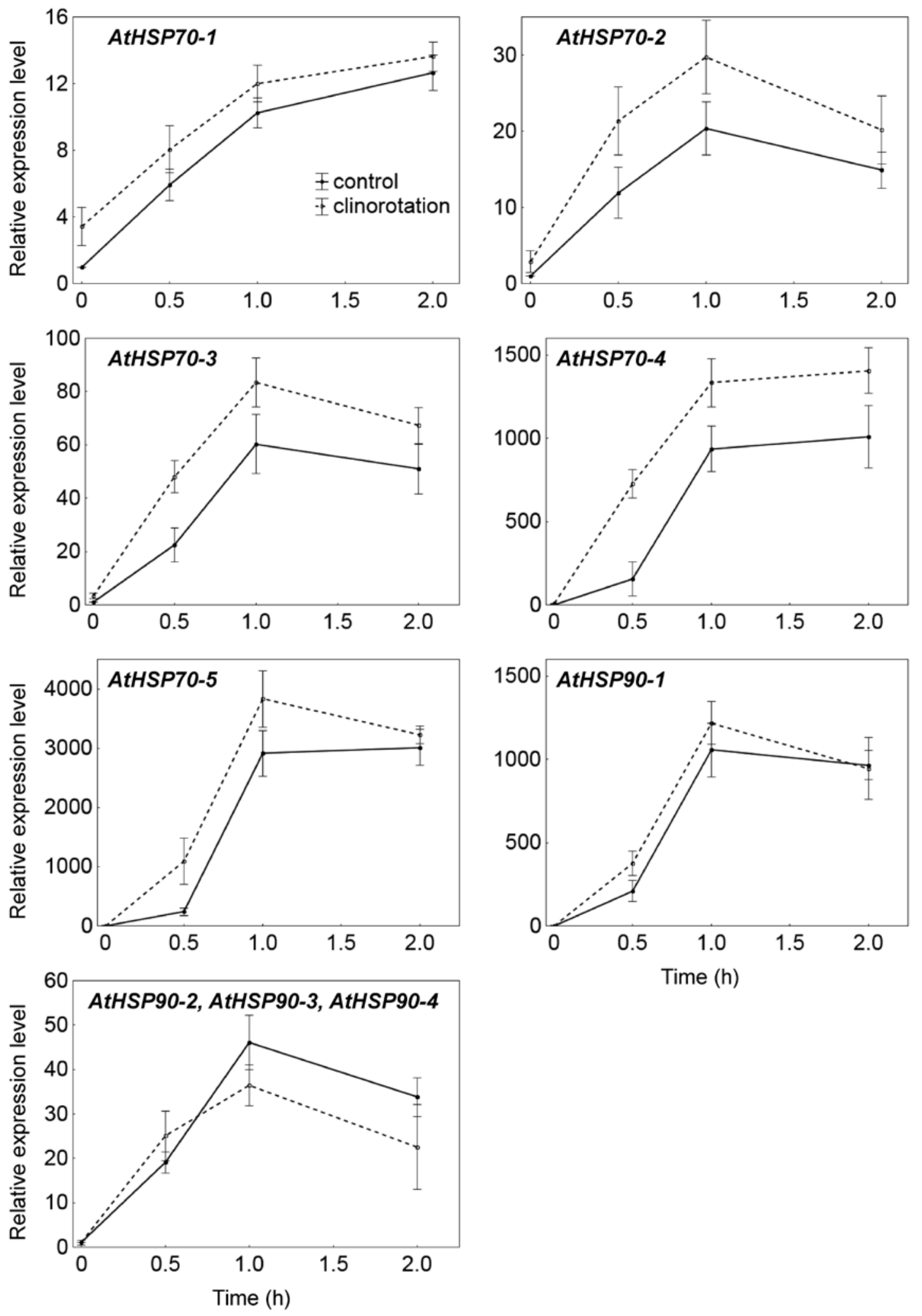

Figure 1. An effect of clinostat rotation on induction of HSP70 and HSP90 genes in Arabidopsis thaliana (Col) seedlings during heat shock. 12-d-old seedlings grown under stationary conditions (control) or clinorotation were exposed at $37^{\circ} \mathrm{C}$ for the indicated times. Expression levels were assessed using RTqPCR, and normalized with respect to UBQ5 mRNA. Relative mRNA amounts were calculated as a fold change to the control ( $=0 \mathrm{~h}$ of the heat treatment). Data are means of three independent biological samples with three analytical replicates \pm s.d. The effect of high temperature was significant for all the HSPs; the effect of clinorotation was significant for AtHSP70-2, AtHSP70-3, AtHSP70-4, AtHSP70-5, and AtHSP90-1 (Two-way ANOVA, p < 0.05). 


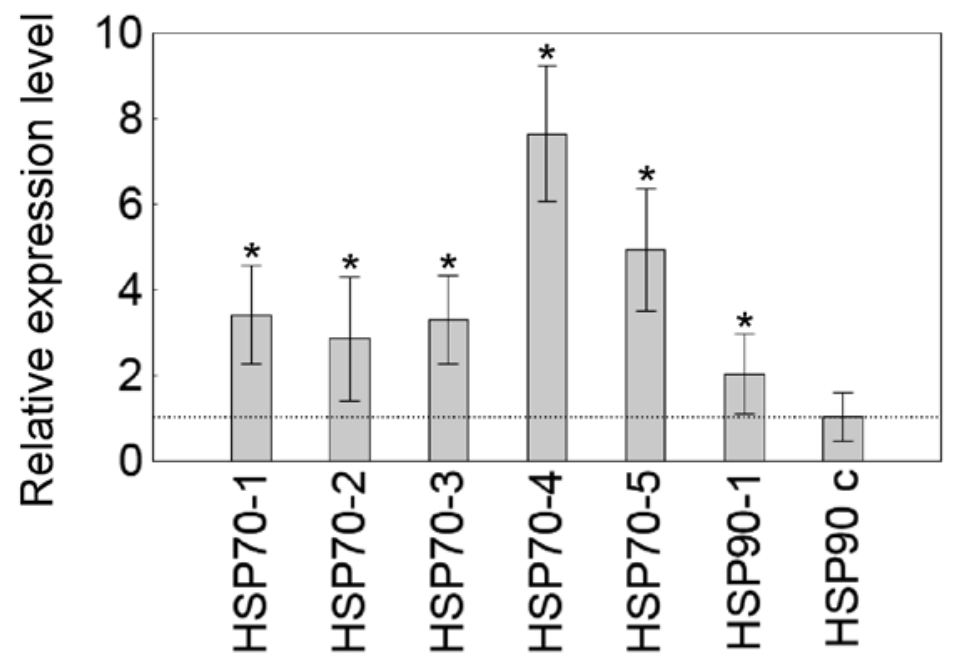

Figure 2. An effect of clinostat rotation on expression of $\mathrm{HSP70}$ and $\mathrm{HSP} 90$ genes in Arabidopsis thaliana (Col) seedlings. The bars present the fold change of transcript abundance in 12-d-old seedlings grown under clinorotation relative to the control (seedlings grown under stationary conditions, = 1; dotted line). HSP90 c represents AtHSP90-2, AtHSP90-3, and AtHSP90-4 (in total). Bars denoted with an asterisk (*) indicate differences between the rotated and control seedlings that are statistically significant, compared with the control (Student's t-test, $p<0.05$ ). Expression levels were assessed using RT-qPCR and normalized with respect to UBQ5 mRNA. Data are means of three independent biological samples with three analytical replicates \pm s.d.
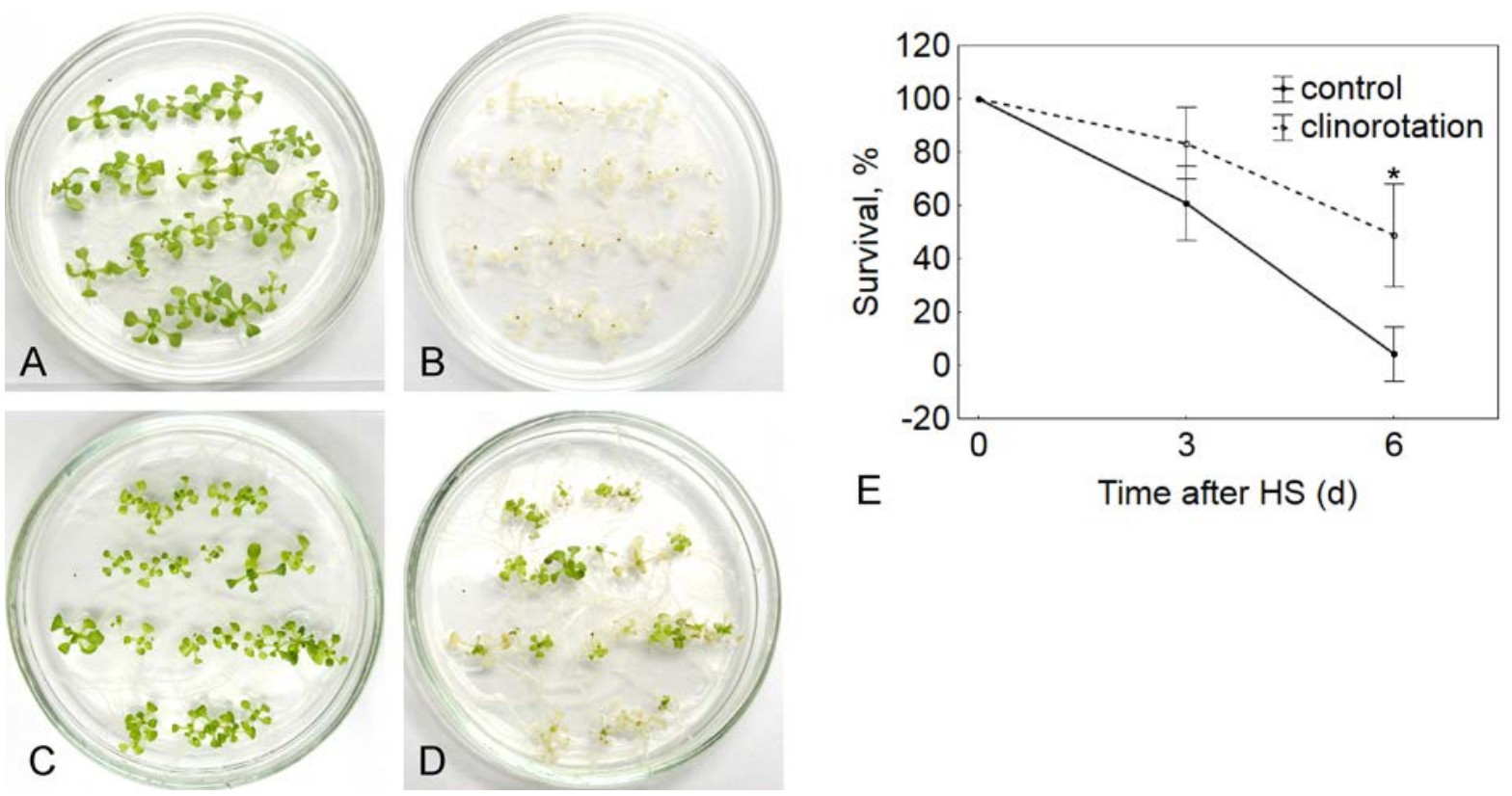

Figure 3. An effect of clinostat rotation on heat shock survival of Arabidopsis thaliana (Col) seedlings. 12d-old seedlings grown under stationary conditions (control) (A, B) or clinorotation (C, D) at $22 \pm 1^{\circ} \mathrm{C}$ were exposed at $45^{\circ} \mathrm{C}$ for $45 \mathrm{~min}$ and recovered under the stationary conditions. $(\mathrm{A}, \mathrm{C})$ seedlings before the heat treatment and $(B, D)$ seedlings after a 6-d recovery period $(B, D)$. (E) Survival rate of the seedlings after the heat treatment. Data are means \pm s.d. $(n=3$ plates with $\sim 25$ seedlings each; * Student's t-test, $\mathrm{p}<0.05)$.

exposure to $45^{\circ} \mathrm{C}$ for $45 \mathrm{~min}$. This temperature is specified as a standard to detect alterations in A. thaliana plant thermotolerance (Silva-Correia et al., 2014). Figure 3 depicts that different survival rates were observed in heat-treated seedlings grown under clinorotation compared with stationary conditions. A portion of rotated seedlings were able to generate new leaves over 
the 6-d post-exposure period, whereas control seedlings showed almost complete death.
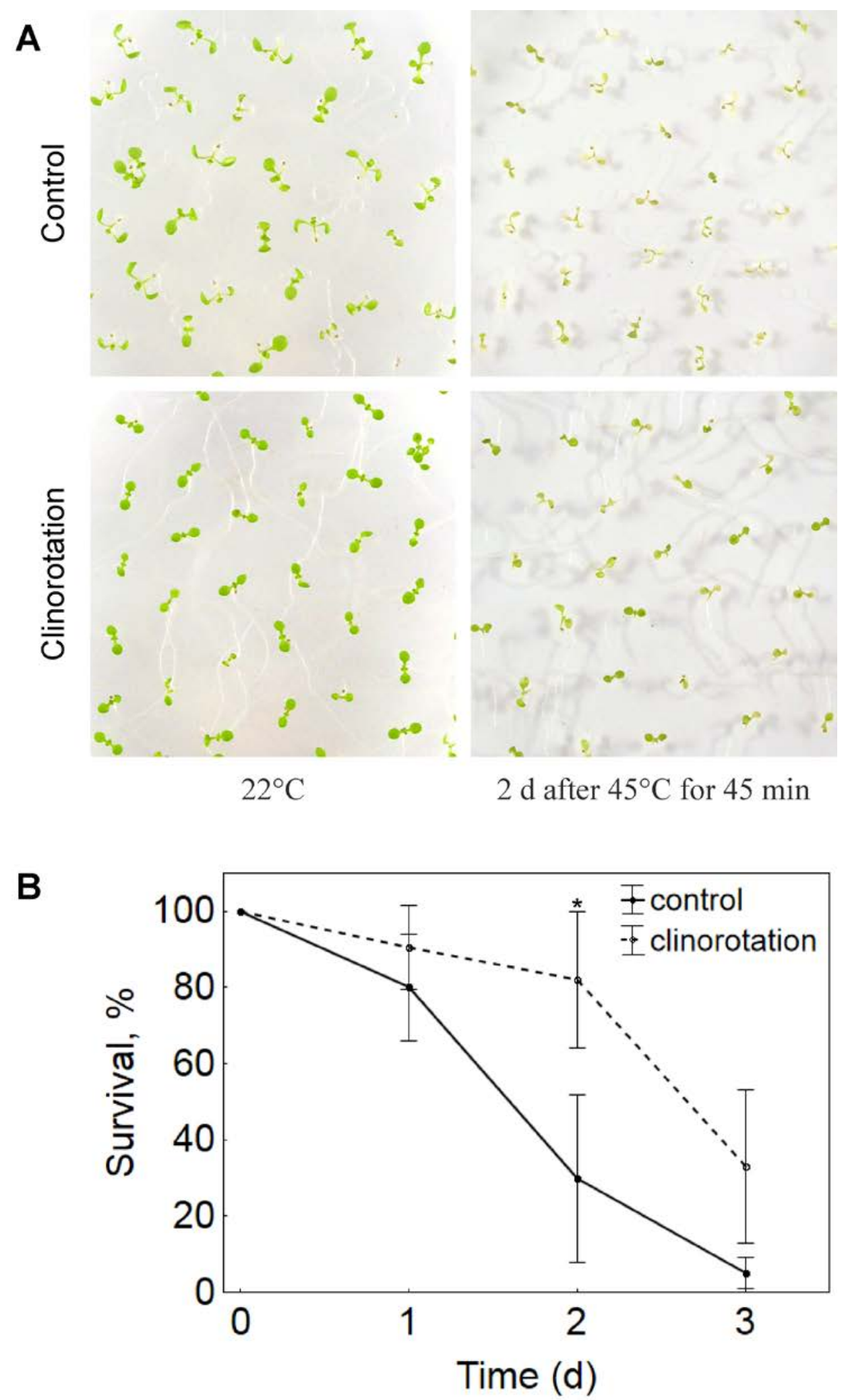

Figure 4. An effect of clinostat rotation on heat shock survival of Arabidopsis thaliana (Col) seedlings. (A) 5 -d-old seedlings grown in the stationary conditions or under clinostat rotation at $22 \pm 1^{\circ} \mathrm{C}$ were exposed at $45^{\circ} \mathrm{C}$ for $45 \mathrm{~min}$, and recovered under the stationary conditions for $3 \mathrm{~d}$. (B) Survival rate of the seedlings after the heat treatment. Data are means \pm s.d. $(\mathbf{n}=3$; * Student's t-test, $\mathbf{p}<0.05)$. 


\section{DISCUSSION}

HSPs/chaperones play a role in protecting cells against multiple stressful conditions (Sørensen et al., 2003; Wang et al., 2004). Their upregulation during the heat shock response provides resumption of protein homeostasis and leads to a higher level of thermotolerance (Vierling, 1991). Moreover, an increasing number of studies demonstrate that HSPs contribute to cross-protection between heat stress and other types of stress in plants (Banti et al., 2008; Montero-Barrientos et al., 2010). In this study, we applied heat treatment following clinorotation of seedlings. As previously mentioned, both factors can activate HSP synthesis. The obtained results showed that clinorotation caused the minor increase in transcript abundance of five cytosolic AtHSP70s and AtHSP90-1 under normal temperature, as well as a faster onset and enhancement of their induction during heat shock. Notably, the strongest effect was revealed for inducible AtHSP70-4, AtHSP70-5, and AtHSP90-1, which are especially important for stress tolerance (Sørensen et al., 2003). In contrast, the total expression of constitutive AtHSP90-2, AtHSP90-3, and AtHSP90-4 was not altered under clinorotation and showed less induction by heat treatment in rotated seedlings, when compared to seedlings grown under stationary conditions.

A weak increase in the expression levels of several HSPs in seedlings grown under clinorotation at normal temperature likely reflects adaptation of cells to rotation. It is noteworthy that clinorotation may have multiple impacts on organisms, including not only simulation of the microgravity effects, but also mechanical and electromagnetic influences, etc. However, in our previous work with pea seedlings, comparison of the effects of horizontal and vertical rotation showed small but statistically significant differences in HSP70 and HSP90 levels that could be attributed to simulated microgravity (Kozeko and Kordyum, 2006).

As a whole, the time course of HSP expression during heat exposure was in good agreement with the previous findings by other researchers (Milioni and Hatzopoulos, 1997; Sung et al., 2001). At that, the magnitudes of AtHSP70s and AtHSP90-1 induction were higher in rotated seedlings relative to control ones. These findings correlate with our previous
Western-blot data indicating enhanced induction of HSP70 and HSP90 in pea seedlings in response to high temperatures $\left(38-45^{\circ} \mathrm{C}\right)$ after long-term clinorotation (Kozeko, 2008). Thus, increasing the heat shock response under the influence of clinorotation was shown at both transcriptional and translational levels. However, the obtained results could indicate two opposite situations. On the one hand, the higher inducibility of several HSPs may reflect higher sensitivity of rotated seedlings to heat shock; on the other hand, it may result from preadaptation of seedlings under clinorotation. To test that, the seedling survival assay was carried out. Its results showed that seedlings grown under clinorotation withstood exposure to the near lethal temperature better than seedlings grown under stationary conditions. These data support the assumption that clinorotation may provide cross-protection of plants against fluctuations in environmental conditions, which correlates with the data on HSP expression.

\section{REFERENCES}

Banti V, Loreti E, Novi G, Santaniello A, Alpi A, Perata P (2008) Heat acclimation and cross-tolerance against anoxia in Arabidopsis. Plant, Cell and Environment 31: 1029-1037

Ferl RJ, Wheeler R, Levine HG, Paul A-L (2002) Plants in space. Current Opinion in Plant Biology 5: 258-263

Hausmann N, Fengler S, Hennig A, FranzWachtel M, Hampp R, Neef M (2014) Cytosolic calcium, hydrogen peroxide and related gene expression and protein modulation in Arabidopsis thaliana cell cultures respond immediately to altered gravitation: parabolic flight data. Plant Biology 16 (Suppl 1): 120-128

Kordyum EL (2014) Plant cell gravisensitivity and adaptation to microgravity. Plant Biology 16 (Suppl 1): 79-90

Kozeko L (2008) Effects of simulated microgravity on thermotolerance of pea seedlings. Journal of Gravitational Physiology 15(1): 173-174

Kozeko L, Kordyum E (2006) The stress protein level under clinorotation in context of the seedling developmental program and the stress response. Microgravity Science and Technology XVIII-3/4: 254-256

Kozeko LY, Kordyum EL (2007) Heat shock proteins Hsp70 and Hsp90 in pea 
seedlings under clinorotation of different duration. Journal of Gravitational Physiology 14(1): 115-116

Lin B, Wang J, Liu H, Chen R, Meyer Y, Barakat A, Delseny M (2001) Genomic analysis of the Hsp70 superfamily in Arabidopsis thaliana. Cell Stress \& Chaperones 6(3): 201-208

Medina FJ, Mancuso S, Hampp R (2011) Plant Sciences. In Laboratory Science with Space Data. Accessing and Using SpaceExperiment Data, D. Beysens, L. Carotenuto, J.J.W.A. van Loon, and M. Zell (eds), pp 117-122. Berlin, Heidelberg: Springer-Verlag

Milioni D, Hatzopoulos P (1997) Genomic organization of hsp90 gene family in Arabidopsis. Plant Molecular Biology 35: 955-961

Montero-Barrientos M, Hermosa R, Cardoza RE, Gutiérrez S, Nicolás C, Monte E (2010) Transgenic expression of the Trichoderma harzianum hsp70 gene increases Arabidopsis resistance to heat and other abiotic stresses. Journal of Plant Physiology 167(8): 659-665

Paul A-L, Popp MP, Gurley WB, Guy C, Norwood KL, Ferl RJ (2005) Arabidopsis gene expression patterns are altered during spaceflight. Advances in Space Research 36: 1175-1181

Paul A-L, Zupanska AK, Ostrow DT, Zhang Y, Sun Y, Li JL, Shanker S, Farmerie WG, Amalfitano CE, Ferl RJ (2012) Spaceflight transcriptomes: unique responses to a novel environment. Astrobiology 12(1): 40-56

Silva-Correia J, Freitas S, Tavares RM, LinoNeto T, Azevedo H (2014) Phenotypic analysis of the Arabidopsis heat stress response during germination and early seedling development. Plant Methods 10(7): 11

Sørensen JG, Kristensen TN, Loeschcke V (2003) The evolutionary and ecological role of heat shock proteins. Ecology Letters 6: 1025-1037

Sung DY, Vierling E, Guy CL (2001) Comprehensive expression profile analysis of the Arabidopsis Hsp70 gene family. Plant Physiology 126: 789-800

Vierling E (1991) The roles of heat shock proteins in plants. Annual Review of Plant Physiology and Plant Molecular Biology 42: $579-620$

Wang W, Vinocur B, Shoseyov O, Altman A (2004) Role of plant heat-shock proteins and molecular chaperones in the abiotic stress response. Trends in Plant Science 9(5): 244-252

Wheeler RM (2010) Plants for human life support in space: from Myers to Mars. Gravitational and Space Biology 23(2): 25-35

Zupanska AK, Denison FC, Ferl RJ, Paul A-L (2013) Spaceflight engages heat shock protein and other molecular chaperone genes in tissue culture cells of Arabidopsis thaliana. American Journal of Botany 100(1): 235-248 